\title{
Secretory Carcinoma of the Breast in an Elderly Woman
}

\section{Aïda Ayadi-kaddour ${ }^{1^{*}}$, Rym Hamrouni ${ }^{1}$, Dorra Zegal $^{2}$ and Faouzi El Mezni ${ }^{2}$}

${ }^{1}$ Department of Pathology, Abderrahmen Mami Hospital, Ariana 2080, Tunisia

2Department of Gynecology and Obstetrics, Maternity and Neonatology, Tunisian Medical Center La Rabta, Tunis, Tunisia

*Corresponding author: Aïda Ayadi-Kaddour, Department of Pathology, Abderrahmen Mami Hospital - 2080 Ariana Tunisia, Tel: 0021622551641 ; E- mail: kaddour1fr@yahoo.fr

Rec date: Mar 29, 2015, Acc date: Apr 19, 2015, Pub date: Apr 21, 2015

Copyright: (c) 2015 Ayadi-kaddour A, et al. This is an open-access article distributed under the terms of the Creative Commons Attribution License, which permits unrestricted use, distribution, and reproduction in any medium, provided the original author and source are credited.

\begin{abstract}
Secretory carcinoma is a very rare and distinctive type of low-grade breast carcinoma, initially termed "juvenile breast cancer", but it is now known to occur in adults of both sexes. It is the only epithelial tumor of the breast characterized by specific translocation. In adults, it is potentially more aggressive than in children.

We report a case of an indolent breast tumor in a 66-years-old woman. The patient presented with a locally advanced mass in the right breast. She had radical mastectomy with axillary node dissection. Grossly, the mass was circumscribed, measured $50 \times 35 \mathrm{~mm}$, with a white glistening cut section. Microscopic examination revealed the classical features of secretory carcinoma with microcystic and solid patterns with abundant intra and extracellular PAS-positive secretory material. The tumor cells showed abundant amphophilic and clear cytoplasm with mildly atypical nuclei. Mitotic figures were infrequent. The axillary node dissection was free of tumor. The patient is disease free after a follow-up period of 12 months.
\end{abstract}

Secretory carcinoma is a very rare type of breast carcinoma and is uncommon in adults. Despite their good behavior, the prognostic factors and the therapeutic management are non-consensual. The majority of patients usually remain free of disease after surgical excision.

Keywords: Secretory carcinoma; Breast; Histology

\section{Introduction}

Secretory carcinoma is a rare low-grade breast carcinoma, characterized by the presence of large amounts of intracellular and extracellular secretory material and composed of cells with granular eosinophilic or amphophilic cytoplasm [1]. This tumor frequently occurs in either children or adolescents [2]. Though it is considered rare in adults, it is potentially more aggressive than in children. Originally this tumor was described in children, but it is now known to occur in adults of both sexes including a substantial number of post-menopausal women [3]. We present the case of a 66-years-old woman with juvenile secretory carcinoma of the breast treated by mastectomy and axillary node dissection.

\section{Case Report}

A 66-year-old female patient presented with a subareolar mass of the right breast since 5 years. The mass suddenly increased in size with no evidence of palpable axillary lymph nodes. Serum tumor markers and other routine blood test were normal. The liver ultrasonography and chest X-ray were negative for metastases. A radical mastectomy with axillary node dissection was decided and performed. Grossly, the mass was circumscribed, measured $50 \times 35 \mathrm{~mm}$ and had white glistening cut section (Figure 1). Microscopic examination revealed the classical features of secretory carcinoma with microcystic, papillary and solid patterns with abundant intra and extracellular PAS-positive secretory material (Figures 2 and 3 ).

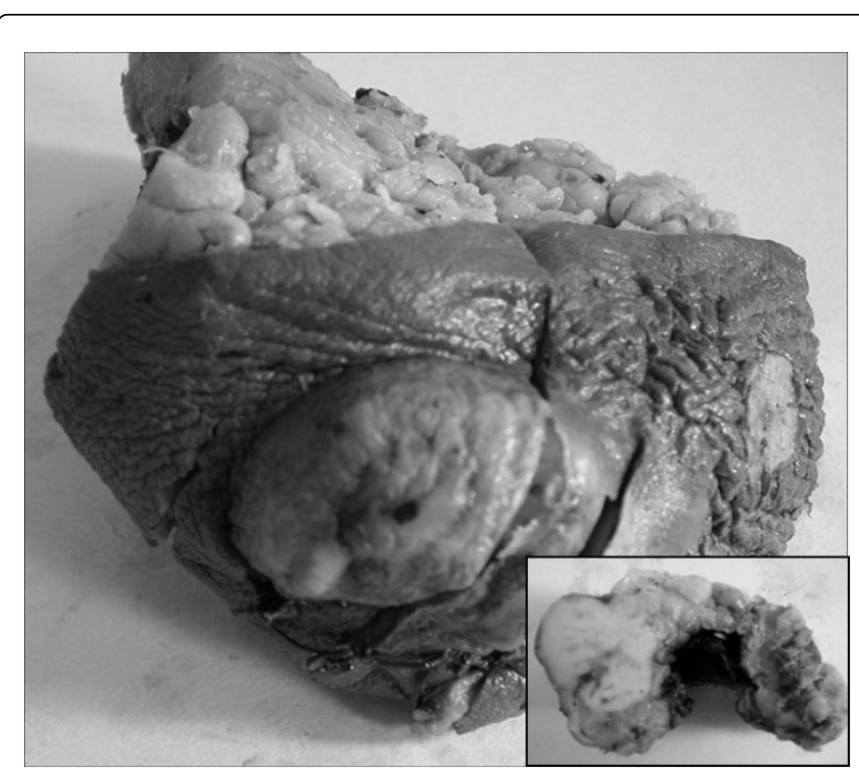

Figure 1: Well circumscribed subareolar mass with white glistening cut section.

The tumor was separated by hyalinising fibrosis and showed partial infiltration of the margin. The tumor cells showed an abundant amphophilic and clear cytoplasm with mildly atypical nuclei (Figure 4). Mitotic figures were infrequent. No tumor infiltration was present at the nipple or at surgical margins. On immunohistochemistry, the 
tumor cells were positive for S100 protein, EMA, keratin, and polyclonal CEA, but negative for progesterone and oestrogen receptor. The tumor was Her2neu negative. The final pathology diagnosis was a secretory carcinoma of $5 \mathrm{~cm}$ of diameter (molecular subtype basal like), grade 2 according to SBR modified. The axillary node dissection was free of tumor. There was no indication for chemotherapy because of the non-responsiveness of this cancer in addition to the absence of node-positive disease. The patient received radiation. After five weeks of radiation therapy, patient's follow-up time has been started, with annual clinical and diagnostic imaging controls. The patient was free from local recurrence or metastases 12 months after surgery.

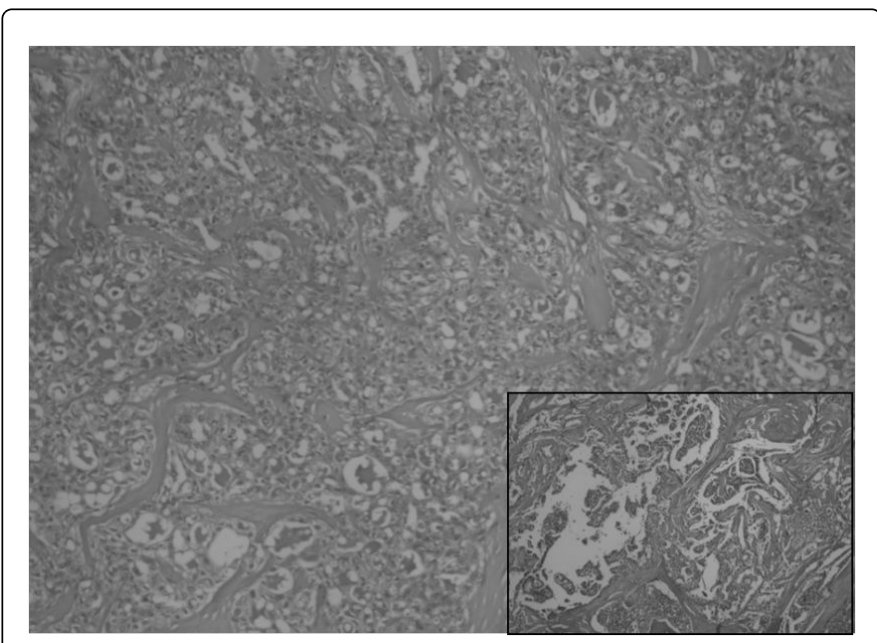

Figure 2: Carcinomatous proliferation with massive, trabecular and papillary architecture

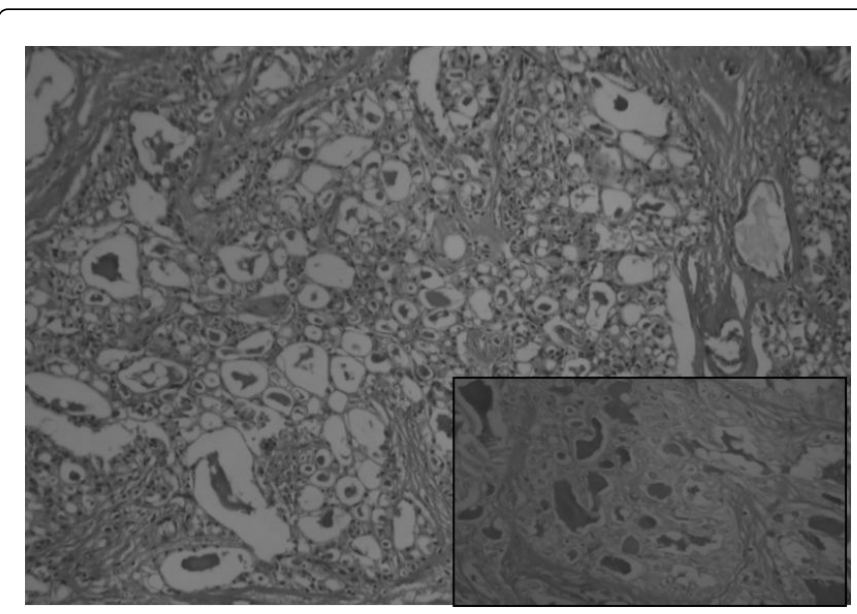

Figure 3: Microcystic and cribriform massive appearance with eosinophilic secretory material PAS+ in the glandular lumen

\section{Discussion}

Secretory carcinoma accounts for significantly less than $0.1 \%$ of all breast carcinomas. It was originally described in 1966 by McDivitt and Stewart who saw seven cases of this carcinoma, all were in children [2], so they designated the tumor as "juvenile" carcinoma.

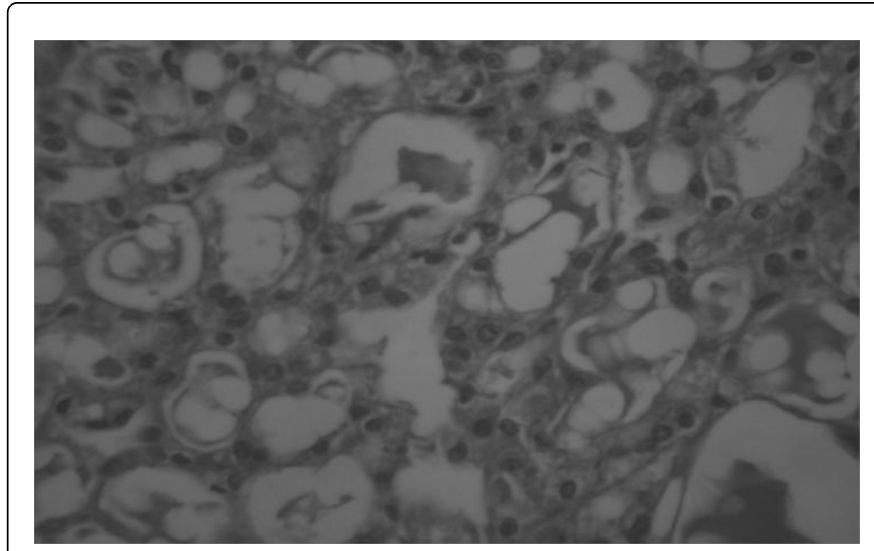

Figure 4: The tumor cells are uniform, with medium size and minimal cytologic atypia

It is most common under the age of 30 , and it is the most common type of breast carcinoma in children. Although originally described in children, it is now known to occur in adults of both sexes, with male to female ratio of $1 / 6$ [1]. The age at presentation varies from 3 to 80 years with a median age of 25 years, therefore, the term secretory carcinoma is preferred. The most frequent clinical presentation is an asymptomatic mobile mass, which is usually subareolar. Multicentric disease was only rarely reported [4]. Since the patient reported that the lesion was present for at least 7 years, it is assumed that it had a slow growing behavior, in an indolent fashion. Mammographically, the tumor has a well-delineated outline with marginal irregularities and calcifications may be present [4]. The tumor size varies from less than 1 to $16 \mathrm{~cm}$ with an average diameter of $3 \mathrm{~cm}$. Larger tumors occur in older patients [5]. Gross tumors are firm and lobulated or circumscribed. The cut surface is solid and varies from gray-white to yellow-taned [6]. Microscopically, secretory carcinomas can demonstrate several histological patterns including, solid, microcystic, and ductal, with many tumors containing all three patterns. The tumor cells are polygonal with typical bubbly or granular eosinophilic cytoplasm due to secretory material [5]. Tubule formation is common and may have secretion in the lumens. Follicular pattern (thyroid-like) may be seen. The secretory material in cells, lumens, and stroma is mucicarmine, alcian blue, and PAS positive, diastase resistant. It is characterized by low-grade nuclear cytology, bland, uniform nuclei, inconspicuous nucleoli and rare mitotic figures [4]. The tumor cells may grow as solid masses separated by fibrous septa, surround minuscule spaces, or form larger cystic spaces. Sheet-like growth with mainly circumscribed margins with occasional foci of infiltration and in situ component are common. Immunohistochemically, tumor cells are EMA, CK, ACE (polyclonal), S-100, and a lactalbumin positive [7]. Most tumors are negative for oestrogen receptor; more than half of the cases are positive for progesterone receptor. Tumors were all negative for Her2neu. The few cases that have been evaluated for immunoexpression of the lysozyme, alpha-1-antitrypsin, have been positive [1]. A single chromosomal translocation manifested by ETV6NTRK3 gene fusion has been proposed as a primary event in the development of secretory carcinoma [8]. Because of its frequently welldelineated nature, secretory carcinoma is often clinically mistaken for a fibroadenoma, particularly when it occurs in adolescent females. Because of the presence of abundant secretory material, frequent circumscription, and occurrence in women of reproductive years, 
secretory carcinoma may also be mistaken for a lactating adenoma. The microscopic appearance however, is quite distinctive [4-7]. Other differential diagnosis includes invasive ductal carcinoma of other types, granular cell tumor, microglandular adenosis and cystic hypersecretory carcinoma [4]. While in many cases, secretory carcinoma behaves as a low-grade cancer, a more aggressive course is manifested in rare patients. The overall incidence of axillary lymph node infiltration is around $30 \%$ in children and adults regardless of gender and recurs after local excision; hence, axillary lymph node dissection is advocated for tumors $\geq 2 \mathrm{~cm}$ [1-6]. Nevertheless, sentinel lymph node biopsy has been used in several studies and has proven to be a useful evaluation method for the lymph node status of secretory breast carcinoma. On the other hand, secretory breast carcinoma is considered to be more aggressive in adult women than in children. For old patients, an initial simple mastectomy with axillary node dissection might be adequate. At our patient, core biopsy of the tumor was not performed, and the advanced age and the size of the tumor made prefer performing dissection. Tavassoli and Norris reported 4 cases of secretory carcinoma in a retrospective series of 7038 breast carcinoma cases and suggested three features of secretory carcinoma that indicate a favorable prognosis : tumor size less than $2 \mathrm{~cm}$, age of less than 20 years at diagnosis, and tumor with circumscribed margins [9]. Carcinomas in men appear to be more aggressive [1]. Recurrences may develop 6 and 8 years following both local excision and modified radical mastectomy, respectively. Distant metastases from secretory carcinoma are extremely rare and were usually reported even after 20 years of surgery [5]. Even though adjuvant therapy has been administered in many cases there is no evidence to support any form of therapy [10]. In our case, a tumor size of $5 \mathrm{~cm}$ and absence of metastasis in axillary nodes suggests a relatively good prognosis. In spite of that, secretory carcinoma being an indolent tumor, a longterm follow-up is required to reach definite conclusions [11]. Surgery is considered the primary treatment of secretory carcinoma [1]. But no published guidelines for surgical management exist. Local recurrences can happen in residual breast tissue or in the chest wall many years after initial surgery. Few studies reported local recurrence in some patients therefore; mastectomy appears to be a sound surgical choice [10]. There are no data on conservative treatment, but this option could be explored particularly in cases where breast development has not yet occurred. In rare cases, systemic metastases have resulted in death of patient [5]. Only 5 cases with distant metastases were reported in the literature, but there are several other reported cases of patients with distant metastases who were treated with either single agent or combination chemotherapy without success [10]. Vindesine, 5-FU, mitomycin, prednisone, adriamycin, epirubicin, cyclophosphamide, carboplatin, and even newer active agents such as docetaxel were some of the drugs used, among others. Postoperative radiotherapy and adjuvant chemotherapy can be used. The prognosis for secretory carcinoma is favorable when compared with ductal carcinoma [11].

\section{Conclusion}

Secretory carcinoma is a very rare type of breast carcinoma with very wide age range of occurrence. This tumor has characteristic morphologic appearance, immunophenotype, and molecular characteristics. Despite their good behavior, the prognostic factors and the therapeutic management are non-consensual.

\section{References}

1. Ozguroglu M, Tascilar K, Ilvan S, Soybir G, Celik V (2005) Secretory carcinoma of the breast. Case report and review of the literature. Oncology 68: 263-268.

2. McDivitt RW, Stewart FW (1966) Breast carcinoma in children. JAMA 195: 388-390.

3. Yaqoob N, Kayani N, ul Hasan SH (2006) Painless breast lump in an elderly woman. Secretory breast carcinoma in an elderly woman. Arch Pathol Lab Med 130: 1073-1074.

4. Li D, Xiao X, Yang W, Shui R, Tu X, et al. (2012) Secretory breast carcinoma: a clinicopathological and immunophenotypic study of 15 cases with a review of the literature. Mod Pathol 25: 567-575.

5. Krausz T, Jenkins D, Grontoft O, Pollock DJ, Azzopardi JG (1989) Secretory carcinoma of the breast in adults: emphasis on late recurrence and metastasis. Histopathology 14: 25-36.

6. Simpson JS, Barson AJ (1969) Breast tumours in infants and children: a 40-year review of cases at a children's hospital. Can Med Assoc J 101: 100-102.

7. Diallo R, Schaefer KL, Bankfalvi A, Decker T, Ruhnke M, et al. (2003) Secretory carcinoma of the breast: a distinct variant of invasive ductal carcinoma assessed by comparative genomic hybridization and immunohistochemistry. Hum Pathol 34: 1299-1305.

8. Tognon C, Knezevich SR, Huntsman D, Roskelley CD, Melnyk N, et al. (2002) Expression of the ETV6-NTRK3 gene fusion as a primary event in human secretory breast carcinoma. Cancer Cell 2: 367-376.

9. Tavassoli FA, Norris HJ (1980) Secretory carcinoma of the breast. Cancer 45: 2404-2413.

10. Herz H, Cooke B, Goldstein D (2000) Metastatic secretory breast cancer. Non-responsiveness to chemotherapy: case report and review of the literature. Ann Oncol 11: 1343-1347.

11. Osako T, Takeuchi K, Horii R, Iwase T, Akiyama F (2013) Secretory carcinoma of the breast and its histopathological mimics: value of markers for differential diagnosis. Histopathology 63: 509-519. 